\title{
Twenty-Five Years of Heart Transplantation at Papworth Hospital: Changes in Factors Influencing Short- and Long-Term Patient Survival Over Time
}

\author{
K.A. Goldsmith, L.D. Sharples*, C. Sudarshan, J. Parameshwar, S. Tsui, J. Wallwork and S. Large \\ Papworth Hospital NHS Trust Research and Development Department, and Cardiothoracic Transplant Units, Papworth \\ Everard, Cambridge, UK, CB23 8RE and MRC Biostatistics Unit, Robinson Way, Cambridge, UK, CB2 2SR
}

\begin{abstract}
: 25 years of heart transplantation data were used to identify factors associated with patient survival and investigate changes over time. Analysis was performed across 5 time eras - pre-triple therapy, post-triple therapy to 1990 and the remaining 15 years through 2005 divided into 3 groups of 5 years each. Both short- and long-term survival improved with the advent of triple therapy, but remained unchanged from the early 1990's. Mean donor and recipient age, proportion of female donors and recipients, transplants with two human leucocyte antigen (HLA)-DR mismatches, ischaemic and cardiopulmonary bypass times (CPB) have increased, while rates of rejection and infection have decreased over time. Female donor and recipient diagnosis were independent predictors of short-term mortality. Older age, recipient diagnosis, 2 or more early rejection episodes and number of HLA-A mismatches were independent predictors of mortality in the longterm. Survival rates after heart transplantation improved with advances in patient care, but have remained static since, during which time there have been increases in risk factors and use of more marginal donors.
\end{abstract}

Key Words: Heart transplantation, changes over time, survival analysis.

\section{INTRODUCTION}

Heart transplantation is a well-established treatment for heart failure [1]. The first heart transplant at our institution was performed in 1979, and the procedure has been routinely done for more than twenty-five years.

The main cause of early mortality after transplant is graft failure [1]. Mortality a year or more after transplant is mostly due to cardiac allograft vasculopathy (CAV) [1], which is thought mainly to result from chronic rejection. Modifications of , and additions to, immunosuppression therapy over time have helped to delay and minimize rejection [2-4]. Since the introduction of cyclosporine in 1982, and of triple therapy (cyclosporine, azathioprine and prednisone administered with antithymocyte globulin [ATG]) in 1986, survival after heart transplantation has improved elsewhere [2-5] and at our institution [6]. Advances in donor organ preservation and diagnosis of rejection have also occurred [7]. The International Society for Heart and Lung Transplantation (ISHLT) report that survival has improved in each 5 year period after 1982-1988, although most of this improvement can be attributed to better early post-operative (6-12 month) survival [1].

Risk factors that have been reported to affect survival after heart transplantation include: donor and recipient age, sex, and cytomegalovirus (CMV) status, recipient diagnosis,

*Address correspondence to this author at the Papworth Hospital NHS Trust Research and Development Department, Papworth Everard, Cambridge, UK, CB23 8RE; Tel: 01480 364445; Fax: 01480 831450;

E-mail: linda.sharples@papworth.nhs.uk donor organ ischaemic time, human leucocyte antigen (HLA) mismatches, donor cause of death, episodes of infection and acute rejection and being on ventricular assist device (VAD) support [1,6;8-20]. A few studies have looked at changes in risk factors over time, and have identified increases in donor and recipient age, female recipients, ischaemic times, and illness and pre-operative inotropic and mechanical support in recipients [1,9;21-23]. These findings indicate that heart transplantation has been performed with more donors and recipients at higher risk (marginal donors and recipients) over time. The concomitant decrease in donors over time [24-28] has led to the use of hearts from borderline donors [22]. It is difficult to delineate the effect more marginal donors and recipients have on survival after heart transplantation when there have been concurrent improvements in donor management and post-transplant treatment [7,29-31].

Studies that have looked at changes in risk factors affecting survival after heart transplantation over time have not combined the use of extensive multiple covariate analyses, survival conditional on surviving the early post-transplant period and a time period as long as 25 years. Using such techniques, this study aims to assess changes in risk factors for survival, and operative and post-operative characteristics, over time and their influence upon survival after heart transplantation. The study was done in particular to see if there were any changes in the impacts of known risk factors and to see whether increases in risk factors in donors and recipients over time had a detrimental effect upon survival. Such information may be useful for centres with shorter follow-up times. 


\section{METHODS}

\section{Subjects}

Between 1979 and December, 2005, there were 1048 first adult heart transplants (97.8\% of all heart recipients) during this period. Donors and recipients were matched on ABO blood type and height. Most transplants were performed using the bi-atrial technique [32]. In the mid-1990's, a donor management and resuscitation protocol was implemented at our institution to increase the donor pool [33,34]. This protocol includes full invasive haemodynamic monitoring to assess function and application of optimized treatment for the donor heart based upon the results of monitoring. Donors are started on thyroxine and vasopressin infusion and blood sugar levels of the donor are carefully monitored and maintained. Recipients are consented for use of their routinely gathered data for research upon acceptance to the heart transplantation waiting list, so patients were not further consented for use of their information in this study.

\section{Variable Definitions}

Short-term survival was defined as patient survival to 3 months post-transplant. Long-term survival was defined as patient survival past 3 months post-transplant. Survival times were calculated from transplantation to death or censoring at 31/12/2005. CMV status was paired as follows: donor negative/recipient negative (D-/R-), donor negative/recipient positive (D-/R+), donor positive/recipient negative (D+/R-), and donor positive/recipient positive $(\mathrm{D}+/ \mathrm{R}+)$. For some analyses, ischaemic and cardiopulmonary bypass (CPB) times were grouped into quartiles. Rejection was defined as grade 3 or above or grade 2 with haemodynamic compromise requiring treatment. Infection was documented when an organism was identified in association with clinical evidence of sepsis.

\section{Statistical Analysis}

Analysis was performed across 5 eras: pre-triple therapy (1979-03/1986), and 4 triple therapy eras: 04/1986-1990; 1991-1995; 1996-2000; 2001-2005. The eras were constructed to separate out the pre-triple therapy era, where outcomes might be expected to be different from those that followed, with the remaining years divided into 5-year time periods, providing groups of a sufficient size for analysis, while attempting to ensure consistency in outcomes within a given era. Changes in recipient and donor characteristics and operative factors were assessed over time using MantelHaenszel test for trend for categorical variables, ANOVA or Kruskal-Wallis tests for continuous variables, the log rank test for trend for long-term survival and Poisson regression and likelihood ratio tests for rejection and infection rates. Time at risk of rejection/infection was calculated as the time between transplantation and death or censoring at 31/12/ 2005.

Logistic and Cox proportional hazards regression models were used to explore the relationships between short- and long-term patient survival, respectively, and the following potential explanatory variables: donor and recipient age, sex and CMV status, recipient diagnosis, donor cause of death, and human leukocyte antigen (HLA) mismatches, while adjusting for transplant era. Rejection and infection episodes in the first three months post-transplant were also included as potential explanatory variables in the analysis of long-term survival. Variables that were not considered for logistic or Cox proportional hazards models are shown in the tables as NC (not considered). Variables that were considered for these analyses, but were not in the final models are shown in the tables as dashes (---). As the time eras served as a proxy for changes over time, including immunosuppression treatment, no further adjustment for type of treatment was made. Backwards stepwise regression methods were used. Variables significant at the $p<0.2$ level in univariate analysis were included in the initial multiple model. The likelihood ratio test was used to determine which variables to remove at each iteration. The variable with the largest $p$-value was removed and this was repeated until those remaining in the model were significant at $p<=0.1$. For the modeling, the significance level used was 0.1. Any variables listed above that were not significant at this level were tested in the final model to see if they were significant at $p<=0.1$. This level was used in order to ensure that all potentially important covariates were included in the models. For the other analyses, a significance level of 0.05 was used.

\section{RESULTS}

Of the 1048 patients undergoing heart transplantation, 23 had a second heart transplant and one had a third transplant in this period. The number of heart transplants peaked in 1987/1988 and then declined.

The most common causes of death within 3 months of transplant were infection (28.8\%), acute rejection (19.9\%, though only 4 patients since 1991), donor organ failure $(16.4 \%)$ and cerebrovascular accident (6.8\%). After 3 months, patients most commonly died from CAV (18.5\%), malignancies $(7.3 \%)$ and infection $(4.2 \%)$.

\section{Changes Over Time}

Changes over time in pre-transplantation risk factors are shown in Table 1 and changes in operative factors and outcomes including survival are shown in Table 2. Mean recipient age increased over the first two eras, then has remained steady since $(p<0.001)$. Mean donor age and ischaemic times have increased $(\mathrm{p}<0.001)$. There was also a higher proportion of female donors $(p=0.022)$, female recipients $(p<0.001)$ and transplants with two HLA-DR mismatches over time $(p=0.015)$. A smaller proportion of ischaemic heart disease (IHD) diagnoses was seen in the most recent era and transplants in patients with rarer conditions increased in the 1996 to 2000 era, with this increase maintained in the 2001-2005 era $(p<0.001)$. The proportion of donors that died from trauma decreased with a concomitant increase in donors that died from stroke, although these changes did not reach significance. In the last two eras, transplants were performed in recipients using VAD support; most of these were in the most recent era. The proportion of transplants with CMV mismatches and two mismatches at the HLA-A and -B loci have not changed significantly over time. $\mathrm{CPB}$ and ventilation times have increased $(p<0.001)$, while stay in ITU varied without any monotonic pattern $(\mathrm{p}<0.001)$. Short-term survival increased with the advent of triple therapy and again in the next era $(\mathrm{p}=0.002)$. Long-term survival in 3-month survivors also improved with triple therapy from $88.7 \%$ to 
Table 1. Changes in Characteristics of Heart Transplants Over Five Eras

\begin{tabular}{|c|c|c|c|c|c|c|}
\hline Factor & Pre-TT & TT-1990 & 1991-1995 & $1996-2000$ & 2001-2005 & p-Value \\
\hline Number of transplants & 150 & 259 & 236 & 220 & 183 & \\
\hline $\mathrm{R}$ age, mean $(\mathrm{SD})$ & $40.0(9.6)$ & $46.6(11.1)$ & $49.0(9.8)$ & $49.3(10.3)$ & $46.8(13.1)$ & $<0.001^{1}$ \\
\hline $\mathrm{R} \operatorname{sex}, \mathrm{n}(\%)$ female & $14(9.3)$ & $35(13.5)$ & $34(14.4)$ & $40(18.2)$ & $42(23.0)$ & $<0.001^{2}$ \\
\hline $\begin{array}{c}\text { R diagnosis, n (\%) IHD } \\
\text { n (\%) dilated CM } \\
\text { n (\%) other }\end{array}$ & $\begin{array}{c}71(47.3) \\
71(47.3) \\
8(5.3)\end{array}$ & $\begin{array}{c}149(57.5) \\
102(39.4) \\
8(3.1)\end{array}$ & $\begin{array}{c}118(50.0) \\
104(44.1) \\
14(5.9)\end{array}$ & $\begin{array}{c}105(47.7) \\
83(37.7) \\
32(14.5)\end{array}$ & $\begin{array}{l}57(31.1) \\
96(52.5) \\
30(16.4)\end{array}$ & $<0.001^{2}$ \\
\hline VAD support n (\%) yes & 0 & 0 & 0 & $7(3.2)$ & $27(14.8)$ & ND \\
\hline $\mathrm{D}$ age, mean $(\mathrm{SD})$ & $24.6(7.6)$ & $28.4(10.6)$ & $34.2(12.2)$ & $35.5(13.1)$ & $37.8(12.8)$ & $<0.001^{1}$ \\
\hline D sex, $\mathrm{n}(\%)$ female & $32(21.3)$ & $80(30.9)$ & $84(35.7)$ & $80(36.4)$ & $59(32.2)$ & $0.022^{2}$ \\
\hline $\begin{array}{c}\text { D cause of death, n }(\%) \text { stroke } \\
\text { n (\%) trauma } \\
\text { n (\%) other }\end{array}$ & $\begin{array}{l}59(39.3) \\
74(49.3) \\
17(11.3)\end{array}$ & $\begin{array}{c}84(34.9) \\
125(51.9) \\
32(13.3)\end{array}$ & $\begin{array}{l}96(48.0) \\
82(41.0) \\
22(11.0)\end{array}$ & $\begin{array}{l}116(58.3) \\
62(31.2) \\
21(10.6)\end{array}$ & $\begin{array}{l}99(55.6) \\
56(31.5) \\
23(12.9)\end{array}$ & $0.90^{2}$ \\
\hline Mean mins ischaemic time (SD) & $160.3(32.2)$ & $165.1(41.9)$ & $186.0(64.0)$ & $195.3(52.4)$ & $217.8(54.6)$ & $<0.001^{1}$ \\
\hline CMV mismatches, $\mathrm{n}(\%) \mathrm{D}+/ \mathrm{R}-$ & $16(12.9)$ & $47(18.2)$ & 44 (18.6) & $45(20.5)$ & $29(16.4)$ & $0.42^{2}$ \\
\hline $\begin{array}{l}\text { HLA-A mismatches, } \mathrm{n}(\%) 0-1 \\
\mathrm{n}(\%) 2\end{array}$ & $\begin{array}{l}83(57.2) \\
62(42.8)\end{array}$ & $\begin{array}{l}160(64.5) \\
88(35.5)\end{array}$ & $\begin{array}{l}83(57.6) \\
61(42.4)\end{array}$ & $\begin{array}{c}135(63.7) \\
77(36.3)\end{array}$ & $\begin{array}{l}98(56.3) \\
76(43.7)\end{array}$ & $0.68^{2}$ \\
\hline $\begin{array}{l}\text { HLA-B mismatches, } \mathrm{n}(\%) 0-1 \\
\mathrm{n}(\%) 2\end{array}$ & $\begin{array}{l}63(43.4) \\
82(56.6)\end{array}$ & $\begin{array}{l}110(44.4) \\
138(55.6)\end{array}$ & $\begin{array}{l}60(41.7) \\
84(58.3)\end{array}$ & $\begin{array}{c}89(42.0) \\
123(58.0)\end{array}$ & $\begin{array}{c}72(41.4) \\
102(58.6)\end{array}$ & $0.55^{2}$ \\
\hline $\begin{array}{l}\text { HLA-DR mismatches, } \mathrm{n}(\%) 0-1 \\
\mathrm{n}(\%) 2\end{array}$ & $\begin{array}{l}70(61.4) \\
44(38.6)\end{array}$ & $\begin{array}{l}141(58.3) \\
101(41.7)\end{array}$ & $\begin{array}{l}82(57.7) \\
60(42.3)\end{array}$ & $\begin{array}{l}108(50.9) \\
104(49.1)\end{array}$ & $\begin{array}{l}86(50.0) \\
86(50.0)\end{array}$ & $0.015^{2}$ \\
\hline
\end{tabular}

${ }^{1}$ ANOVA p-value, ${ }^{2}$ Mantel-Haenszel test for trend p-value, $\mathrm{R}=$ recipient, $\mathrm{D}=$ donor, $\mathrm{TT}=$ triple therapy, $\mathrm{SD}=$ standard deviation, $\mathrm{IHD}=\mathrm{ischaemic}$ heart disease, $\mathrm{CM}=\mathrm{cardiomyopathy}$, $\mathrm{VAD}=$ ventricular assist device. $\mathrm{ND}=$ not calculated as not available for all eras.

Table 2. Changes in Operative Factors and Outcomes after Heart Transplantation Over Five Eras

\begin{tabular}{|c|c|c|c|c|c|c|}
\hline Factor & Pre-TT & TT-1990 & 1991-1995 & 1996-2000 & 2001-2005 & p-Value \\
\hline Number of transplants & 150 & 259 & 236 & 220 & 183 & \\
\hline Median mins CPB time (IQR) & $94(27)$ & $84(30)$ & $102(33)$ & $114(40)$ & $122(54)$ & $<0.001^{1}$ \\
\hline Median hrs ventilation (IQR) & $7.0(5.8)$ & $10.0(6.0)$ & $7.0(3.3)$ & $9.0(6.0)$ & $12.0(17.0)$ & $<0.001^{1}$ \\
\hline Median days in ITU (IQR) & $6.5(5.0)$ & $3.0(2.0)$ & $2.0(1.0)$ & $1.0(1.0)$ & $3.0(5.0)$ & $<0.001^{1}$ \\
\hline 3-month survival $n, \%$ alive & $115(76.7)$ & $220(84.9)$ & $211(89.4)$ & $192(87.3)$ & $164(89.6)$ & $0.002^{2}$ \\
\hline Survival conditional on 3-month survival at 1 year & 88.7 & 94.1 & 95.3 & 93.2 & 94.5 & $0.001^{3}$ \\
\hline Survival conditional on 3 -month survival at 5 years & 73.0 & 77.7 & 84.4 & 84.4 & --- & \\
\hline $\begin{array}{c}\text { Rejection episodes within } 3 \text { mo, } \mathrm{n}(\%) 0 \\
\mathrm{n}(\%) 1 \\
\mathrm{n}(\%) 2 \text { or more }\end{array}$ & $\begin{array}{l}31(20.7) \\
60(40.0) \\
59(39.3)\end{array}$ & $\begin{array}{l}72(27.8) \\
91(35.1) \\
96(37.1)\end{array}$ & $\begin{array}{l}80(33.9) \\
79(33.5) \\
77(32.6)\end{array}$ & $\begin{array}{l}86(39.1) \\
65(29.5) \\
69(31.4)\end{array}$ & $\begin{array}{l}115(62.8) \\
46(25.1) \\
22(12.0)\end{array}$ & $<0.001^{2}$ \\
\hline $\begin{array}{c}\text { Infection episodes within } 3 \mathrm{mo}, \mathrm{n}(\%) 0 \\
\mathrm{n}(\%) 1 \\
\mathrm{n}(\%) 2 \text { or more }\end{array}$ & $\begin{array}{l}69(46.0) \\
52(34.7) \\
29(19.3)\end{array}$ & $\begin{array}{l}162(62.5) \\
58(22.4) \\
39(15.1)\end{array}$ & $\begin{array}{c}149(63.1) \\
69(29.2) \\
18(7.6)\end{array}$ & $\begin{array}{l}151(68.6) \\
45(20.5) \\
24(10.9)\end{array}$ & $\begin{array}{c}162(88.5) \\
14(7.7) \\
7(3.8)\end{array}$ & $<0.001^{2}$ \\
\hline
\end{tabular}

${ }^{1}$ Kruskal-Wallis p-value, ${ }^{2}$ Mantel-Haenszel test for trend $\mathrm{p}$-value, ${ }^{3}$ Log-rank test for trend $\mathrm{p}$-value, TT $=$ triple therapy, $\mathrm{CPB}=$ cardiopulmonary bypass, IQR=interquartile range.

$94.1 \%$ at 1 year $(\mathrm{p}=0.001)$. However, both short- and longterm survival remain unchanged from the early 1990's to date. The proportion of patients with two or more rejection and infection episodes within 3 months of transplantation has decreased over time $(\mathrm{p}<0.001)$. 


\section{Infection and Rejection Rates}

Treated rejection rates have decreased over time early after transplantation (Fig. 1, p<0.001), and between 4 to 6 months post-transplantation $(\mathrm{p}=0.006)$. Infection rates have decreased over time in all 3 month periods post-transplantation up to 1 year (Fig. 1, p <0.001). Between 7 and 12 months post-transplantation, there was a decrease in infection rates from pre-triple therapy to the first triple therapy era, which has been maintained in subsequent eras.
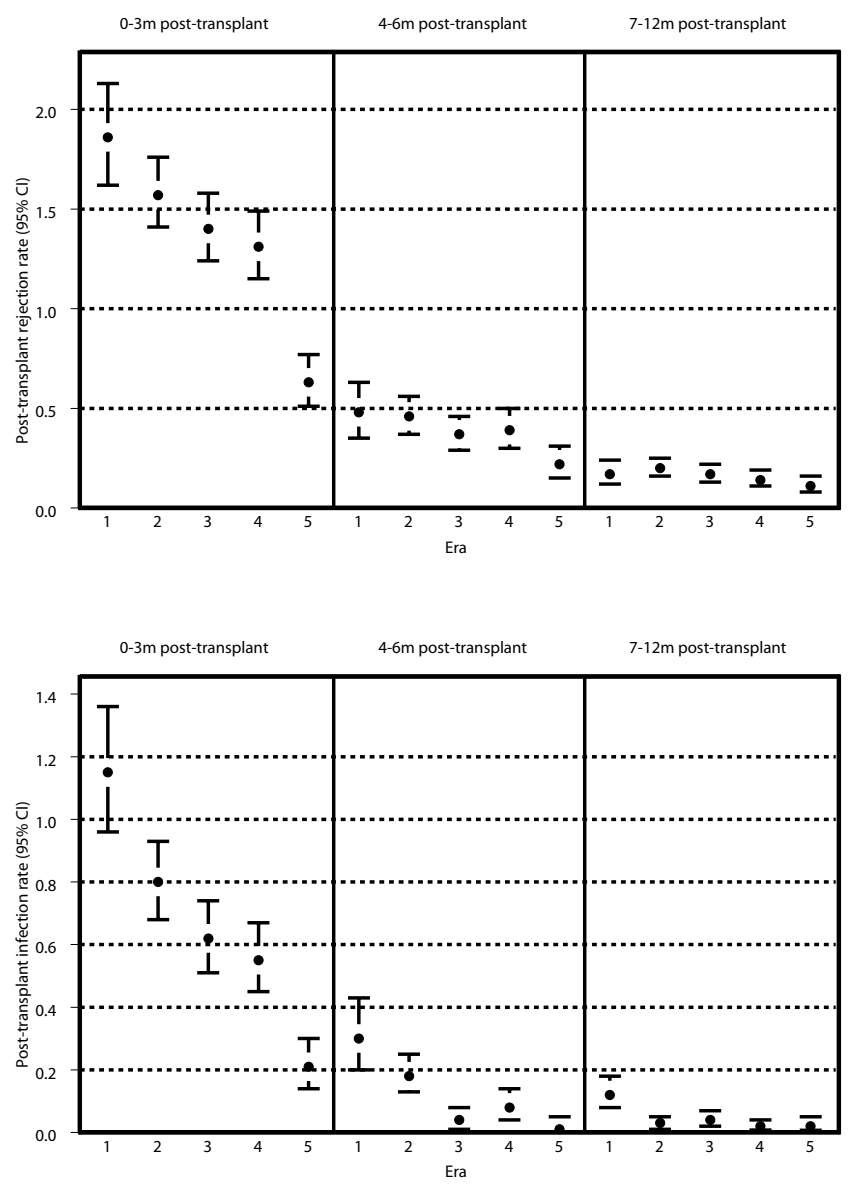

Fig. (1). Post-transplant rejection and infection rates per 100 patient days.

\section{Short-Term Survival}

Results are summarized in Table 3. Transplant recipients in the pre-triple therapy era were more likely to die within 3 months than those in the most recent era. Female recipients, and those with female donors, were more likely to die in the short-term. In general, greater CPB times increased the likelihood that an individual died early. Recipients with a diagnosis of IHD, or with other diagnoses were more likely to die within 3 months than people with dilated cardiomyopathy (CM). Receiving a heart from a CMV positive donor, especially when the recipient was CMV negative, was related to death early after transplantation. There was a weak relationship between longer ischaemic times and dying within 3 months, although this was not significant in the presence of other variables. Recipient and donor age, HLA mismatches and donor cause of death were not associated with short-term mortality in this study.
Multiple logistic regression analysis showed that donor sex and recipient diagnosis were the strongest predictors of short-term mortality when adjusting for transplant era (Table 3).

\section{Long-Term Survival}

Survival (including the first three months post-transplantation) for the cohort of transplant recipients after 1, 5, 10 and 20 years were $80.5 \%, 69.3 \%, 54.1 \%$ and $23.4 \%$. The same figures for transplant recipients conditional on surviving 3 months were $93.6 \%, 80.5 \%, 62.9 \%$ and $27.2 \%$ (Fig. 2). The median survival time from first transplant for this subgroup was 13.0 years (95\% CI, $11.7-14.3$ ).

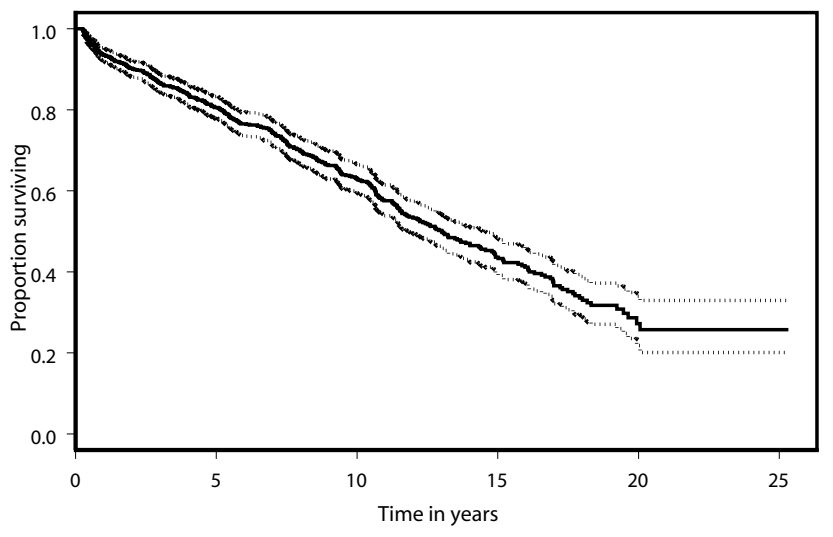

Fig. (2). Survival from 1st transplant by year in recipients surviving for 3 months.

Results of the Cox regression analysis are summarized in Table 4. Older recipients and recipients of organs from older donors had poorer long-term survival. Longer ventilation time and IHD diagnosis were also significantly associated with poorer long-term survival. Having one HLA-A mismatch was weakly associated with better long-term survival when compared to having no mismatches, although this did not reach significance. Recipients of hearts from CMV positive donors fared worse than those receiving hearts from CMV negative donors, particularly CMV negative recipients. Recipient sex, donor sex, ischaemic time, CPB time, HLA-B or -DR mismatches and donor cause of death were not associated with long-term survival.

Multiple Cox regression analysis indicated that recipient age, diagnosis, number of rejection episodes and number of HLA-A mismatches were the strongest predictors of longterm survival when adjusting for transplantation era (Table 4).

\section{DISCUSSION}

The existence of more than 25 years of heart transplantation experience provided an opportunity both to observe changes in patient care over time and to update our knowledge of the characteristics of recipients and donors and how these affect outcomes. This is particularly important in light of the shortage of donors in order to determine the effect of expansion of donor selection criteria and improvements in treatment over time on recipient outcomes. 
Table 3. Relationship between Pre-Transplant and Operative Characteristics and Short-Term (3 Month) Mortality

\begin{tabular}{|c|c|c|c|c|}
\hline Risk Factor & $\begin{array}{c}\text { Died } \\
n=146\end{array}$ & $\begin{array}{c}\text { Survived } \\
\mathbf{n}=902\end{array}$ & $\begin{array}{l}\text { OR Adjusted for } \\
\text { Era (95\% CI) }\end{array}$ & $\begin{array}{l}\text { OR Multiple }{ }^{1} \\
(95 \% \text { CI })\end{array}$ \\
\hline Mean R age (SD) (OR for $10 \mathrm{y}$ ) & $47.1(11.5)$ & $46.8(11.2)$ & $1.13(0.95,1.34)$ & --- \\
\hline $\begin{array}{c}\text { R sex, } \mathrm{n}(\%) \text { female } \\
\text { ref }=\text { male }\end{array}$ & $30(20.5)$ & $135(15.0)$ & $1.63(1.04,2.55)$ & --- \\
\hline $\begin{array}{c}\text { R diagnosis, } \mathrm{n}(\%) \text { IHD } \\
\mathrm{n}(\%) \text { other } \\
\text { ref = dilated } \mathrm{CM}\end{array}$ & $\begin{array}{l}79(54.1) \\
17(11.6)\end{array}$ & $\begin{array}{c}421(46.7) \\
75(8.3)\end{array}$ & $\begin{array}{l}1.51(1.02,2.22) \\
2.08(1.12,3.87)\end{array}$ & $\begin{array}{l}1.57(1.07,2.32) \\
2.08(1.11,3.87)\end{array}$ \\
\hline $\begin{array}{c}\text { D sex, } \mathrm{n}(\%) \text { female } \\
\text { ref }=\text { male }\end{array}$ & $63(43.2)$ & $272(30.2)$ & $1.93(1.34,2.78)$ & $1.97(1.36,2.85)$ \\
\hline $\begin{array}{l}\text { D cause of death } \\
\text { n (\%) trauma } \\
\text { n (\%) other } \\
\text { ref=stroke }\end{array}$ & $\begin{array}{l}55(39.9) \\
16(11.6)\end{array}$ & $\begin{array}{l}344(41.4) \\
99(11.9)\end{array}$ & $\begin{array}{l}0.85(0.57,1.26) \\
0.90(0.50,1.63)\end{array}$ & --- \\
\hline $\begin{array}{l}\text { Ischaemic time } \\
\mathrm{n}(\%) 149-180 \mathrm{~m} \\
\mathrm{n}(\%) 181-219 \mathrm{~m} \\
\mathrm{n}(\%) 220-393 \mathrm{~m} \\
\mathrm{ref}=18-148 \mathrm{~m}\end{array}$ & $\begin{array}{l}43(30.1) \\
25(17.5) \\
40(28.0)\end{array}$ & $\begin{array}{l}218(24.5) \\
229(25.7) \\
216(24.2)\end{array}$ & $\begin{array}{l}1.24(0.76,2.03) \\
0.78(0.45,1.36) \\
1.71(0.99,2.93)\end{array}$ & $\begin{array}{l}--- \\
--- \\
---\end{array}$ \\
\hline 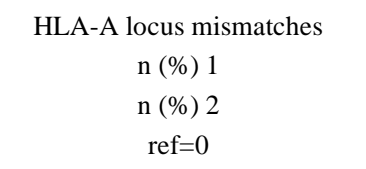 & $\begin{array}{l}63(47.4) \\
54(40.6)\end{array}$ & $\begin{array}{l}393(49.7) \\
310(39.2)\end{array}$ & $\begin{array}{l}0.83(0.46,1.51) \\
0.92(0.50,1.69)\end{array}$ & $\begin{array}{l}--- \\
---\end{array}$ \\
\hline 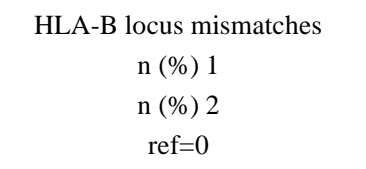 & $\begin{array}{l}44(33.1) \\
84(63.2)\end{array}$ & $\begin{array}{l}301(38.1) \\
445(56.3)\end{array}$ & $\begin{array}{l}1.27(0.47,3.38) \\
1.66(0.64,4.34)\end{array}$ & $\begin{array}{l}--- \\
---\end{array}$ \\
\hline 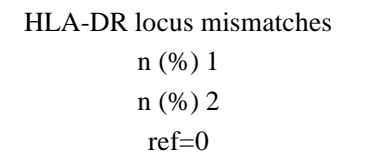 & $\begin{array}{l}51(42.1) \\
58(47.9)\end{array}$ & $\begin{array}{l}372(48.9) \\
337(44.3)\end{array}$ & $\begin{array}{l}0.58(0.29,1.16) \\
0.76(0.38,1.51)\end{array}$ & $\begin{array}{l}--- \\
---\end{array}$ \\
\hline $\begin{array}{c}\text { CPB time, } \mathrm{n}(\%) 86-102 \mathrm{~m} \\
\mathrm{n}(\%) 103-125 \mathrm{~m} \\
\mathrm{n}(\%) 126-207 \mathrm{~m} \\
\mathrm{ref}=25-85 \mathrm{~m}\end{array}$ & $\begin{array}{l}39(30.0) \\
22(16.9) \\
43(33.1)\end{array}$ & $\begin{array}{l}209(23.9) \\
222(25.4) \\
202(23.1)\end{array}$ & $\begin{array}{l}1.93(1.11,3.33) \\
1.21(0.64,2.26) \\
2.95(1.67,5.23)\end{array}$ & $\mathrm{NC}$ \\
\hline
\end{tabular}

${ }^{1} \mathrm{OR}$ adjusted for all variables in final model, $\mathrm{R}=$ recipient, $\mathrm{D}=\mathrm{donor}$, OR=odds ratio, $\mathrm{SD}=$ standard deviation, IHD=ischaemic heart disease, $\mathrm{CM}=\mathrm{cardiomyopathy}$, $\mathrm{CPB}=$ cardiopulmonary bypass, $\mathrm{NC}=$ not considered for multiple models, $---=$ considered but not in final model. 
Table 4. Relationship between Pre-Transplant and Operative Characteristics and Long-Term Survival

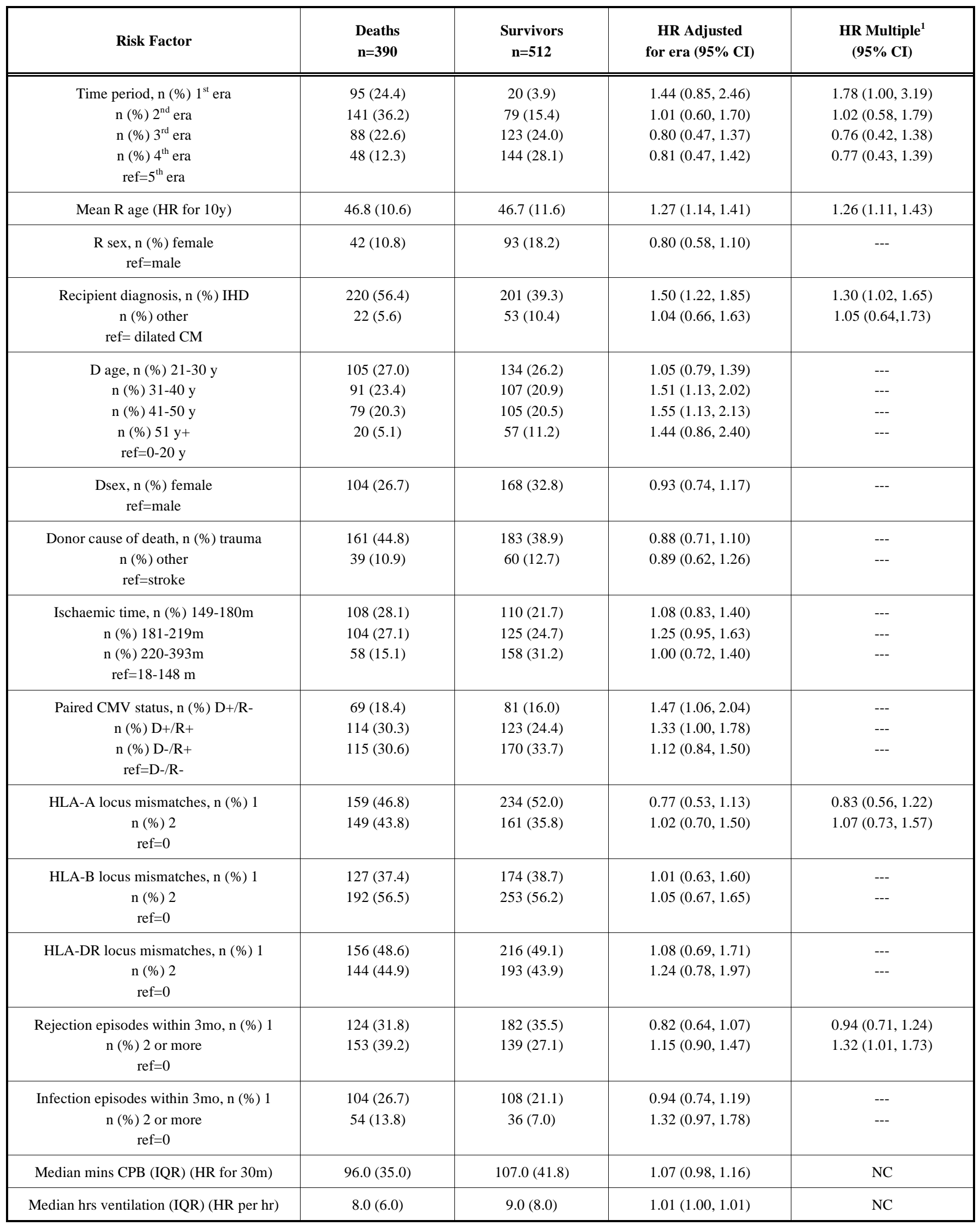

${ }^{1} \mathrm{HR}$ adjusted for all variables in final model, $\mathrm{R}=$ recipient, $\mathrm{D}=$ donor, $\mathrm{HR}=$ hazard ratio, $\mathrm{IHD}=$ ischaemic heart disease, $\mathrm{CM}=$ cardiomyopathy, $\mathrm{CPB}=$ cardiopulmonary bypass, $\mathrm{IQR}=$ interquartile range, $\mathrm{NC}=$ not considered for multiple models, --- = considered but not in final model. 
At our institution, overall patient survival after heart transplantation was similar to that seen by others $[1,12,17$, 19,21-23,35], as were trends in early mortality over time [21]. Despite improvement with the advent of triple therapy, survival has remained static in subsequent time periods. The number of heart transplants performed at our institution per year has generally declined after 1988 . This stems from less availability of donor organs [24-28], probably due in part to the passing of seatbelt legislation in the late 1980s and again in the late 1990s, reflected by fewer donors dying from trauma, and improvements in treatment for stroke. As might be expected, fewer donors and the implementation of the marginal donor resuscitation protocol (see Methods - Subjects section) led to the use of more marginal donors, who were older, more likely to be female and had longer cold ischaemic times in more recent eras. Longer ischaemic times have resulted from fewer domino transplants and more outof-zone donors [36]. Transplants were also performed in recipients with more risk factors; over time recipients were older, more often female, had rarer conditions and were more often using VADs, the latter indicating that some recipients may have been less well acutely, but were stabilized. Similar trends have been seen by others $[1,22]$. Operations at our institution have become more complex over time with longer $\mathrm{CPB}$ and ventilation times. Use of marginal donors and more complex operations in marginal recipients could explain why survival rates have not appreciably improved over time. We did not, however, see a decrease in either short- or long-term survival. Heart transplantation has been shown to be beneficial in the case of high risk recipients and marginal donors [37]. A U.S. study showed improved survival over time despite increasing risk factors for transplantation [22]. Advances in immunosuppression and management of infection and rejection, reflected in these findings by decreasing rejection and infection rates over time, may have counterbalanced the increased risks.

Donor gender and recipient diagnosis predicted early mortality; recipients of hearts from female donors or with rarer diagnoses had nearly two times the odds of early death. Others have also found donor gender to be a predictor of early mortality $[12,22]$ and an association between female donor and worse graft survival [38]. Female recipient also predicted an increased risk of death in the short term. Female recipients most often get female hearts because of sizematching of donor and recipient. Hearts from females tend to be smaller and to generally have a lower power output, which could affect early outcomes. Another possible reason for the risk of early death could be the association of hearts from female donors with early death from acute rejection $[6,10]$. While they were related to short-term survival, donor and recipient gender were not important predictors of longterm survival, which has been seen by others $[10,35,39,40]$. Recipient characteristics were important for long-term survival; the hazard of death for recipients increased 1.3 times for every 10 year increment of age and recipients with IHD diagnosis had 1.3 times the hazard of dying compared to those with dilated CM. This suggests that recipient age and diagnosis should continue to be considered when deciding on suitability for transplant. It has, however, been shown that many older recipients have good outcomes [41-43]. The effect of HLA-A mismatches is not clear and it is likely that acute rejection episodes provide a more direct measure of the effect of tissue mismatch on survival. A detrimental effect of no mismatches on longer term graft survival has been noted before [18]. Rejection remains an important contributor to mortality in the long-term. Although the proportion of recipients having 2 or more rejection episodes within 3 months of transplantation has decreased significantly over time, multiple early rejection episodes increased the risk of death in the long-term, possibly due to acute rejection episodes predisposing these patients to CAV [44]. There is a continued need to understand the mechanisms of rejection and to improve its treatment.

Some characteristics were associated with survival in univariate, but not multiple variable analyses. This does not necessarily mean that these variables are not important predictors of survival in the context of changes over time and variables such as age, gender and diagnosis which are related; multiple variable analysis identifies the most powerful variables and the variables with independent associations with the outcome. For example, CMV matching status was associated with short- and long-term survival in univariate, but not multiple variable analyses. CMV prophylaxis has changed over time; initially none was available, then intravenous prophylaxis with gangcylovir was introduced in 1992 [45], followed by effective oral therapies [46]. Such improvements in CMV prophylaxis and, in common with all infections, decreasing CMV infection over time (data not shown) may explain the weaker effect of donor/recipient CMV mismatch on survival. Donor age was also associated with long-term survival in univariate, but not multiple variable analyses. The effect of donor age on long-term survival found here is consistent with the association between donor age and the onset of CAV, likely due to older donors having the potential to carry pre-existing coronary disease [47].

Although mean donor organ ischaemic time has increased over time, and had a weak association with short-term mortality, it was not an independent predictor of short- or longterm survival in this study. There was some evidence in our study that patients with the longest ischaemic times had poorer short-term survival than those with the shortest when taking era into account, however, this effect did not remain when donor sex and recipient diagnosis were included. A UK study of first adult heart transplants also found an association with short-term mortality in a univariate analysis [36]. Some groups have found a relationship between ischaemic time and 1 year mortality in studies of larger numbers of patients [1,22], but there does not seem to be a relationship with long-term survival $[1,12,22]$. Smaller studies such as ours have less power to detect differences in ischaemic time. It may also be that improvements in donor management have partly overcome increasing ischaemic times. In addition, the effect of ischaemic time is difficult to separate from other risk factors because the transplant decision is multifactorial.

In conclusion, the early increase in survival after cardiac transplantation due to improved immunosuppression and patient care has been maintained but has not increased further. Likely causes are increasing numbers of marginal donors (as a result of the shortage of ideal donors) and higherrisk recipients. Heart transplantation is an extremely effective therapy for patients with end-stage heart disease and strategies to maintain and encourage organ donation are important. 


\section{REFERENCES}

[1] Taylor DO, Edwards LB, Boucek MM, et al. Registry of the International Society for Heart and Lung Transplantation: twenty-third official adult heart transplantation report 2006. J Heart Lung Transplant 2006; 25(8): 869-79.

[2] Bolman R3, Saffitz J. Early postoperative care of the cardiac transplantation patient: routine considerations and immunosuppressive therapy. Prog Cardiovasc Dis 1990; 33(3): 137-48.

[3] Bolman RM3, Olivari MT, Saffitz J, et al. Current results with triple therapy for heart transplantation. Transplant Proc 1987; 19(1 Pt 3): 2490-1.

[4] Modry DL, Oyer PE, Jamieson SW, et al. Cyclosporine in heart and heart-lung transplantation. Can J Surg 1985; 28(3): 274-80.

[5] Kriett JM, Kaye MP. The Registry of the International Society for Heart and Lung Transplantation: eighth official report 1991. J Heart Lung Transplant 1991; 10(4): 491-8.

[6] Sharples LD, Caine N, Mullins P, et al. Risk factor analysis for the major hazards following heart transplantation--rejection, infection, and coronary occlusive disease. Transplant 1991; 52(2): 244-52.

[7] Ellman PI, Ronson RS, Kron IL. Modern concepts in heart transplantation. J Long Term Eff Med Implants 2003; 13(6): 465-83.

[8] Aziz T, Burgess M, Rahman AN, et al. Cardiac transplantation for cardiomyopathy and ischemic heart disease: differences in outcome up to 10 years. J Heart Lung Transplant 2001; 20(5): 525-33.

[9] De Santo LS, Amarelli C, Romano G, et al. Evolving practice patterns in heart transplantation: a single-center experience over 15 years. Transplant Proc 2004; 36(3): 627-30.

[10] Fabbri A, Bryan AJ, Sharples LD, et al. Influence of recipient and donor gender on outcome after heart transplantation. J Heart Lung Transplant 1992; 11(4 Pt 1): 701-7.

[11] Gupta D, Piacentino V3, Macha M, et al. Effect of older donor age on risk for mortality after heart transplantation. Ann Thorac Surg 2004; 78(3): 890-9.

[12] John R, Rajasinghe H, Chen JM, et al. Impact of current management practices on early and late death in more than 500 consecutive cardiac transplant recipients. Ann Surg 2000; 232(3): 302-11.

[13] Pedotti P, Mattucci DA, Gabbrielli F, et al. Analysis of the complex effect of donor's age on survival of subjects who underwent heart transplantation. Transplantation 2005; 80(8): 1026-32.

[14] Radovancevic B, Konuralp C, Vrtovec B, et al. Factors predicting 10-year survival after heart transplantation. J Heart Lung Transplant 2005; 24(2): 156-9.

[15] Sharples LD, Caine N, Mullins P, et al. Analysis of survival data after transplantation and its risk factors for graft loss. Transplant Proc 1991; 23(1 Pt 2): 1167-9.

[16] Shiba N, Chan MC, Valantine HA, et al. Longer-term risks associated with 10-year survival after heart transplantation in the cyclosporine era. J Heart Lung Transplant 2003; 22(10): 1098-106.

[17] Smits JM, Vanhaecke J, Haverich A, et al. Three-year survival rates for all consecutive heart-only and lung-only transplants performed in Eurotransplant, 1997-1999. Clin Transpl 2003: 89-100.

[18] Taylor CJ, Smith SI, Sharples LD, et al. Human leukocyte antigen compatibility in heart transplantation: evidence for a differential role of HLA matching on short- and medium-term patient survival. Transplantation 1997; 63(9): 1346-51.

[19] Topkara VK, Cheema FH, Kesavaramanujam S, et al. Effect of donor age on long-term survival following cardiac transplantation. J Card Surg 2006; 21(2): 125-9.

[20] Tsai FC, Marelli D, Bresson J, et al. Recent trends in early outcome of adult patients after heart transplantation: a single-institution review of 251 transplants using standard donor organs. Am J Transplant 2002; 2(6): 539-45.

[21] John R, Rajasinghe HA, Chen JM, et al. Long-term outcomes after cardiac transplantation: an experience based on different eras of immunosuppressive therapy. Ann Thorac Surg 2001; 72(2): 440-9.

[22] Kirklin JK, Naftel DC, Bourge RC, et al. Evolving trends in risk profiles and causes of death after heart transplantation: a ten-year multi-institutional study. J Thorac Cardiovasc Surg 2003; 125(4): $881-90$.
JAMA 1986; 255(14): 1892-8.

26] Gore SM, Cable DJ, Holland AJ. Organ donation from intensive care units in England and Wales: two year confidential audit of deaths in intensive care. BMJ 1992; 304(6823): 349-55.

[27] Goudarzi BM, Bonvino S. Critical care issues in lung and heart transplantation. Crit Care Clin 2003; 19(2): 209-31.

[28] Westaby S. Surgical restoration of the failing left ventricle. Med Clin North Am 2003; 87(2): 523-52.

[29] Jamieson RW, Friend PJ. Organ reperfusion and preservation. Front Biosci 2008; 13: 221-35.

[30] Kutsogiannis DJ, Pagliarello G, Doig C, et al. Medical management to optimize donor organ potential: review of the literature. Can J Anaesth 2006; 53(8): 820-30.

[31] Poston RS, Griffith BP. Heart transplantation. J Intensive Care Med 2004; 19(1): 3-12.

[32] Lower RR, Shumway NE. Studies on orthotopic homotransplantation of the canine heart. Surg Forum 1960; 11: 18-9.

[33] Potter CD, Wheeldon DR, Wallwork J. Functional assessment and management of heart donors: a rationale for characterization and a guide to therapy. J Heart Lung Transplant 1995; 14(1 Pt 1): 59-65.

[34] Wheeldon DR, Potter CD, Jonas M, Wallwork J, Large SR. Using "unsuitable" hearts for transplantation. Eur J Cardiothorac Surg 1994; 8(1): 7-9.

[35] Edwards NM, Rajasinghe HA, John R, et al. Cardiac transplantation in over 1000 patients: a single institution experience from Columbia University. Clin Transpl 1999; 15: 249-61.

[36] Hussey JC, Bonser RS, Rudge CJ, et al. Ischaemia time in cardiac transplantation: A UK national study. J Heart Lung Transplant 2006; 25(2S): 115

[37] Luckraz H, Sharples LD, Charman SC, et al. Does heart transplantation confer survival benefit in all risk groups? J Heart Lung Transplant 2005; 24(9): 1231-4.

[38] Zeier M, Dohler B, Opelz G, et al. The effect of donor gender on graft survival. J Am Soc Nephrol 2002; 13(10): 2570-6.

[39] De Santo LS, Marra C, De Feo M, et al. The impact of gender on heart transplantation outcomes: a single center experience. Ital Heart J 2002; 3(7): 419-23.

[40] Loebe M, Potapov EV, Hummel M, et al. Medium-term results of heart transplantation using older donor organs. J Heart Lung Transplant 2000; 19(10): 957-63.

[41] Everett JE, Djalilian AR, Kubo SH, et al. Heart transplantation for patients over age 60. Clin Transplant 1996; 10(6 Pt 1): 478-81.

[42] Fabbri A, Sharples LD, Mullins P, et al. Heart transplantation in patients over 54 years of age with triple-drug therapy immunosuppression. J Heart Lung Transplant 1992; 11(5): 929-32.

[43] Fonarow GC. How old is too old for heart transplantation?. Curr Opin Cardiol 2000; 15(2): 97-103.

[44] Sharples LD, Jackson CH, Parameshwar J, et al. Diagnostic accuracy of coronary angiography and risk factors for post-hearttransplant cardiac allograft vasculopathy. Transplantation 2003; 76(4): 679-82.

[45] Wreghitt TG, Abel SJ, McNeil K, et al. Intravenous ganciclovir prophylaxis for cytomegalovirus in heart, heart-lung, and lung transplant recipients. Transpl Int 1999; 12(4): 254-60.

[46] Devyatko E, Zuckermann A, Ruzicka M, et al. Pre-emptive treatment with oral valganciclovir in management of CMV infection after cardiac transplantation. J Heart Lung Transplant 2004; 23(11): 1277-82.

[47] Stoica SC, Cafferty F, Pauriah M, et al. The cumulative effect of acute rejection on development of cardiac allograft vasculopathy. J Heart Lung Transplant 2006; 25(4): 420-5. 